\title{
In Vitro Assessment of the Antioxidant Properties of Aqueous Byproduct Extracts of Vitis vinifera
}

\section{Roberto Puglisi ${ }^{1 *}$, Alex Severgnini', Aldo Tava² and Marina Montedoro'}

'Istituto Sperimentale Italiano Lazzaro Spallanzani, Loc. La Quercia, 26027 Rivolta d'Adda (CR), Italy ${ }^{2}$ CREA-ZA Centro di Ricerca Zootecnia e Acquacoltura, Viale Piacenza 29, 26900 Lodi, Italy

Received: 1 June 2018 Accepted: 31 January 2019

\author{
*Corresponding author: \\ Phone: +39036378883 \\ Fax: +390363371021 \\ E-mail: roberto.puglisi@istitutospallanzani.it
}

ORCID IDs: 0000-0002-5832-5970

(Puglisi), 0000-0002-6894-8071

(Severgnini), 0000-0003-4657-

-4053 (Tava), 0000-0002-8461-0396

(Montedoro)

\section{SUMMARY}

Aqueous extracts were obtained at low temperature with the Naviglio technology from grapevine stalks (Merlot), marc (Merlot and Cabernet Sauvignon) and leaves (Merlot) as typical byproducts of winemaking industry, and their properties were evaluated cytofluorometrically on human dermal fibroblasts. Leaf extracts had the greatest total phenolic ((47.6 \pm 3.5$)$ $\mathrm{mg} / \mathrm{g})$ and proanthocyanidin $((24.2 \pm 0.1) \mathrm{mg} / \mathrm{g})$ contents compared to the others. The preliminary colorimetric MTT (3-[4,5-dimethylthiazole-2-yl]-2,5-diphenyltetrazolium bromide) assay individuated two consecutive non-toxic volume fractions of each extract (from 0.8 to 12.8 $\%)$ that were adopted for three cytofluorometric tests. The first cell membrane test did not evidence any harmful effects against plasma membranes at the two non-toxic volume fractions. The second mitochondrial membrane test showed a decreased $(p<0.01)$ percentage of cells ((15.7 \pm 8.3$)$ vs $(32.5 \pm 1.3) \%$ ) with active polarized mitochondrial membranes at the higher non-cytotoxic volume fractions of extracts from Cabernet Sauvignon marc in response to $4.5 \mathrm{mM} \mathrm{H}_{2} \mathrm{O}_{2}$, and from Merlot stalks ( $\left.\mathrm{p}<0.05\right)$ at $1.5 \mathrm{mM} \mathrm{H}_{2} \mathrm{O}_{2}((49.3 \pm 6.1)$ vs $(64.6 \pm 2.4) \%)$ and without $\mathrm{H}_{2} \mathrm{O}_{2}((89.7 \pm 2.4)$ vs $(96.9 \pm 1.8) \%)$, compared to the controls submitted to the same $\mathrm{H}_{2} \mathrm{O}_{2}$ concentration. Conversely, mitochondrial activity of leaf extracts significantly ( $p<0.05)$ increased ((96.3 \pm 1.8$)$ and $(96.4 \pm 1.4) \%)$ after treatment with $0.5 \mathrm{mM} \mathrm{H}_{2} \mathrm{O}_{2}$ at both non-cytotoxic volume fractions compared to control ((88.2 21.1$) \%)$. Finally, as evidenced by the third oxidative status test, stalk extracts did not evidence relevant effects on the cellular oxidative state, while the extracts of marc and leaves demonstrated significantly medium $(p<0.05)$ to highly $(p<0.001)$ positive effects following exposure to $\mathrm{H}_{2} \mathrm{O}_{2}$ ranging from 0.5 to $4.5 \mathrm{mM}$, compared to controls.

Key words: non-cytotoxic effect, extraction at low temperature, human dermal fibroblasts, oxidative status, mitochondrial membrane

\section{INTRODUCTION}

Grapevine (Vitis vinifera) contains several classes of active components, which have been extensively documented (1). The fruits represent the first source of extraction of its active molecules while the leaves are left on the ground. The majority of byproducts of winemaking and distillery industries, such as stalks, skin, pulp and seeds, often generate waste disposal concerns despite their potential as low-cost source of bioactive ingredients (2). In particular, pomace is very rich in phenolics, and thus, greatly appreciated as natural additive in the food and cosmetic industry (3). However, given the high-quality market standards, comprehensive approaches targeted at their exploitation are required for the assessment of their safety and merits. In this regard, the concept of the 3 Rs - replacement, reduction and refinement, guides the rules for improving the use of cytotoxicity methods as an alternative to animal testing (4). This principle promotes the use of in vitro approaches to investigate the biological activities of drugs at the cellular level. In this respect, the versatility and continuous progress in flow cytometry make this technology a valid tool for detailed analysis of cellular material (5).

Solvent-based technologies involving mixtures of water and organic solvents are often used to recover molecules from solid matrices on the basis of their polar nature (6). In order to be economically advantageous, the choice of the more appropriate solvents is crucial also in 
consideration of their environmental impacts. At the same time, although high temperatures increase extraction efficiency, the stability and integrity of derived compounds may be compromised. Alternative methods entail the use of supercritical fluids. The process appears to be safe for preserving the integrity of molecules of interest compared to traditional organic solvent extraction. However, the correct identification of supercritical fluids, the sample preparation and extraction conditions drastically affect the yield and quality of extraction. An alternative pressurized dynamic extraction process for the recovery of phytocomplexes from solid matrices has been designed (7). The so-called Naviglio Extractor allows for the recovery of several classes of molecules through a series of pressure/depressure cycles, whose effect is to transfer analytes from the solid matrix to the liquid phase due to the negative pressure gradient between the liquid within the solid phase and the solution. The process can be accomplished using any desired solvent in order to prompt the extraction of preferred molecules. Furthermore, the possibility to operate at low temperatures prevents the damaging of thermolabile compounds.

On these bases, the aim of this work is to obtain aqueous extracts at low temperature from grapevine byproducts with the Naviglio technology and to cytofluorometrically evaluate their safety in vitro and antioxidant properties in view of potential enhancements in areas related to cosmetics.

\section{MATERIALS AND METHODS}

\section{Materials and collection of plant matrices}

Chemicals were purchased from Sigma Aldrich, Merck (Rome, Italy), unless otherwise specified. Different matrices of Vitis vinifera were collected in a winery of Franciacorta, northern Italy, from September to early November 2015: (i) Merlot stalks, (ii) Merlot marc and (iii) Cabernet Sauvignon marc were recovered as byproducts of the winemaking process, and (iv) Merlot leaves were harvested in the vineyard of the same company. Samples were stored refrigerated under vacuum. In the laboratory, samples were brought to $-80^{\circ} \mathrm{C}$ and lyophilized using the Alpha 1-4 LSC (Martin Christ GmbH, Osterode am Harz, Germany) under $32 \mathrm{~Pa}$ pressure, with a shelf temperature of $-20^{\circ} \mathrm{C}$ for 5 $\mathrm{h}$ followed by $48 \mathrm{~h}$ at $20^{\circ} \mathrm{C}$. In order to obtain $100 \mathrm{~g}$ lyophilizate, 150,400 and $200 \mathrm{~g}$ of stalks, marc and leaves were used, respectively. Samples were then stored under vacuum until extraction.

\section{Extraction}

Lyophilized material was submitted to solid-liquid extraction using the Naviglio Extractor (Atlas Filtri Engineering, Padua, Italy). Repeated extractions were pursued for $24 \mathrm{~h}$ using $200 \mathrm{~g}$ lyophilizate of each of the four plant matrices dissolved in $2 \mathrm{~L}$ of slightly acidified osmotic water ( $2 \%$ citric acid and $0.1 \%$ potassium sorbate) under the following conditions: 12 piston strokes in the dynamic phase and 900000 reiterating cycles of static (4 min at approx. $10^{6} \mathrm{~Pa}$ ) and dynamic (60 $\mathrm{s}$ at atmospheric pressure) phases.

\section{Evaluation of phenolic content}

Aqueous extracts were assessed (three replicates) to estimate total phenols and proanthocyanidins using a double beam Lambda 35 UV/Vis spectrophotometer (Perkin-Elmer, Milan, Italy). Total phenolics were determined according to the Folin-Ciocalteu method (8) using 100- $\mu \mathrm{L}$ aliquots mixed with $100 \mu \mathrm{L}$ of $50 \%$ Folin-Ciocalteu reagent and $2 \mathrm{~mL}$ of 2.5 $\%(\mathrm{~m} / \mathrm{V})$ sodium carbonate solution. After $2 \mathrm{~h}$, the absorbance was read at $750 \mathrm{~nm}$. Quantitation was based on standard curve of gallic acid $\left(R^{2}=0.9999\right)$ and results were expressed in $\mathrm{mg}$ gallic acid equivalents per $\mathrm{mL}$ of solution. Total proanthocyanidins were determined by the butanol/HCl method (9) using $500-\mu \mathrm{L}$ aliquots mixed with $3 \mathrm{~mL}$ butanol/ $\mathrm{HCl}$ (95:5, by volume) solution in screw capped tubes and incubated for $60 \mathrm{~min}$ at $95^{\circ} \mathrm{C}$. The absorbance was then read at $550 \mathrm{~nm}$. Quantitation was based on standard curve of delphinidin $\left(\mathrm{R}^{2}=0.9989\right)$ and results were expressed in $\mathrm{mg}$ delphinidin equivalents per $\mathrm{mL}$ of solution. Results were then expressed as dry mass of lyophilizates.

\section{In vitro cytotoxicity test}

First, the aqueous extracts were assayed by the MTT (3-[4,5-dimethylthiazole-2-yl]-2,5-diphenyltetrazolium bromide) test to detect potential cytotoxicity in order to establish an appropriate safe dosage to cells. To this aim, early passage human dermal fibroblasts (Caltag Medsystems, Buckingham, UK) were cultured in 100-mm Petri dishes in $12 \mathrm{~mL}$ Dulbecco's modified Eagle's medium (DMEM) supplemented with $10 \%$ fetal bovine serum, penicillin (100 Ul/mL) and streptomycin $(100 \mu \mathrm{g} / \mathrm{mL})$ at $37{ }^{\circ} \mathrm{C}$ in $5 \% \mathrm{CO}_{2}$ humidified atmosphere. At $80 \%$ confluence, cells were harvested by trypsinization and propagated ( $<10$ times). For analysis, cells were seeded into 96-well plates (AGC Techno Glass, Tokyo, Japan) at 10000 cells in $100 \mu \mathrm{L}$ per well and grown for 2 days. Thereafter, cells were incubated for $24 \mathrm{~h}$ in the presence of increasing doses of aqueous extracts as follows: 0 (control), 0.2, 0.4, 0.8, 1.6, $3.2,6.4,12.8,25$ and $50 \%$ (by volume). Cell viability was assessed with the Cell Proliferation Kit I (Roche, Madison, WI, USA). Briefly, $10 \mu \mathrm{L}$ of the labelling reagent were added to each well and incubated for $4 \mathrm{~h}$. Thereafter, $100 \mu \mathrm{L}$ of the solubilization buffer were added and incubated overnight. The absorbance at $490 \mathrm{~nm}$ was recorded using the VersaMax ELISA microplate reader (Molecular Devices, San Jose, CA, USA), and results were expressed as the percentage variation with respect to control cells (100\%). The test included three replicates on different days using four samples per replicate.

Based on the results, two consecutive non-toxic doses (i.e. those volume fractions that induce a decrease in cell viability of less than $20 \%$ compared to control) were individuated for each extract and further adopted for the cytofluorimetric evaluation. The two volume fractions were referred to as low and high doses. 


\section{In vitro cytofluorometric evaluation}

Analyses were conducted using the microcapillary flow cytometer (Guava ${ }^{\circledR}$ EasyCyte $^{\mathrm{TM}}$ Plus, Merck Millipore) as specifically described for each test. Cells were processed as follows: fibroblasts were seeded in 35-mm dishes at 150000 cells/dish in $2 \mathrm{~mL}$ DMEM and left undisturbed for two days until confluence. A single Petri dish at this stage constituted an experimental sample. On the 3rd day of culture the medium was renewed with the same DMEM containing the two (low and high) doses of extracts as detailed below, while controls were prepared by simply renewing the medium. Cells were then cultured at $37^{\circ} \mathrm{C}$ for $24 \mathrm{~h}$.

In order to verify that the extracts were not harmful to cell membranes, a specific cell membrane test was performed as follows: after $24 \mathrm{~h}$ of culture with the extracts, cells were trypsinized, centrifuged (PK121R; ALC International Srl, Cologno Monzese, Italy) for $5 \mathrm{~min}$ at $500 \times \mathrm{g}$ in DMEM and the pellet was suspended in $200 \mu \mathrm{L}$ of the same medium for cytofluorometric analysis. Two positive controls were included by treating control cells for 2 min with a detergent capable of inducing cell Iysis (Reagent S100; ChemoMetec, Allerød, Denmark) at two volume fractions (1 and $3 \%$ ).

For two successive mitochondrial membrane and oxidative status tests, after culturing for $24 \mathrm{~h}$ with the extracts, the cells were treated for $30 \mathrm{~min}$ with five increasing concentrations (0, 0.5, 1.5, 3.0 and $4.5 \mathrm{mM}$ ) of $\mathrm{H}_{2} \mathrm{O}_{2}$ (Scharlab, S.L., Barcelona, Spain) by replacing the medium with $\mathrm{H}_{2} \mathrm{O}_{2}$ supplemented medium. The cells were then harvested and suspended in $200 \mu \mathrm{L}$ (mitochondrial membrane test) or $400 \mu \mathrm{L}$ (oxidative status test) of DMEM for cytometric analysis. For each test, the assessments were repeated during three different days, analyzing at least 5000 cells per assay in duplicate. The analyses were conducted by adapting the procedures implemented for sperm analysis (10).

\section{Cell membrane}

The assessment was performed using double staining. To this aim, the fluorescent dye Sybr ${ }^{\oplus} 14$ (LIVE/DEAD ${ }^{\text {TM }}$ viability kit L7011-A; Invitrogen, Thermo Fisher Scientific, Rodano, Italy), which permeates intact plasmatic membranes, was used to stain nuclear and mitochondrial DNA of both living and dead cells (green emission peak at $516 \mathrm{~nm}$ ). The second dye, propidium iodide (PI; LIVE/DEAD ${ }^{\mathrm{TM}}$ viability kit L7011-B; Invitrogen), is a fluorochrome specific for nucleic acids, which is not able to permeate intact membranes. In the presence of damaged membranes, the dye penetrates into the cell and increases fluorescence 20 times once it intercalates the DNA (red emission peak at $617 \mathrm{~nm}$ ). For analysis, $10 \mu \mathrm{L}$ cell suspension and $188 \mu \mathrm{L}$ DMEM were poured into Eppendorf tubes, stained with $1 \mu \mathrm{L}$ Sybr $14(20 \mu \mathrm{M})$ and $1 \mu \mathrm{L} \mathrm{PI}(24 \mu \mathrm{M})$, and incubated for $10 \mathrm{~min}$ at $37^{\circ} \mathrm{C}$. Thereafter, the tubes were centrifuged for $5 \mathrm{~min}$ at $500 \times g$, the pellets were suspended in $200 \mu \mathrm{L}$ phosphate-buffered saline (PBS) and loaded into a 96-wells plate (polystyrene microplate 650101; Greiner Bio-One GmbH, Kremsmünster, Austria) for analysis.

\section{Mitochondrial membrane}

A $10-\mu \mathrm{L}$ suspension of treated cells was poured into a well of a microplate (Greiner Bio-One $\mathrm{GmbH}$ ), diluted with $189 \mu \mathrm{L}$ of DMEM and $1 \mu \mathrm{L}$ of the fluorescent dye 5,5',6,6'-tetrachloro1,1',3,3'-traethylbenzimidazolylcarbocyanine iodide (JC-1; Invitrogen; $153 \mu \mathrm{M}$ dissolved in dimethyl sulfoxide (DMSO)), and incubated for $15 \mathrm{~min}$ at $37^{\circ} \mathrm{C}$. The results of analysis were expressed as percentage of cells with mitochondria with highly polarized membranes $\left(\Delta \Psi_{m}\right)$ (high yellow-orange fluorescence at $590 \mathrm{~nm}$ ) with respect to the total population, which included the cells with low or without polarized membranes (green fluorescence at $527 \mathrm{~nm}$ ).

\section{Oxidative status}

Oxidative status was evaluated using the dye dihydro- 2 '- -7 'dichlorofluorescein diacetate (H2DCF-DA), which is able to penetrate the cells where it is esterified by endogenous esterases to a non-fluorescent form (H2DCF). In the presence of intracellular reactive oxygen species (ROS), H2DCF is oxidized to the fluorescent dichlorofluorescein (DCF), which can be quantified by monitoring the increase in fluorescence (517-527 nm) proportional to ROS. To this aim, $198 \mu \mathrm{L}$ of treated cells were put in 1.5-mL Eppendorf tube. Thereafter, $2 \mu \mathrm{L}$ of H2DCF-DA (Invitrogen; $1 \mathrm{mM}$ dissolved in DMSO) were added and incubated for $30 \mathrm{~min}$ at $37^{\circ} \mathrm{C}$. Then, $1 \mathrm{~mL}$ PBS was added and centrifuged for $5 \mathrm{~min}$ at $500 \times \mathrm{g}$. After removing the supernatant, the pellet was suspended in $200 \mu \mathrm{L}$ PBS and transferred to a microwell (Greiner Bio-One GmbH) for cytofluorometric analysis.

\section{Statistical analysis}

For membrane integrity and mitochondrial activity, control and sample groups were compared using the Chi square test, while the Student's test was used for comparing groups relative to oxidative status.

\section{RESULTS AND DISCUSSION}

\section{Chemical composition and cytotoxicity}

The extracts obtained from leaves contained the highest quantity of phenolics compared to the others (Table 1). A lower content of total phenolics and proanthocyanidins was detected in the extracts of Merlot marc than in Cabernet Sauvignon marc and stalks.

Table 1. Phenolic composition of aqueous extracts of four grapevine byproducts obtained by a solid-liquid pressurized dynamic extraction using Naviglio ${ }^{\oplus}$ technology

\begin{tabular}{lcc} 
& \multicolumn{2}{c}{$w /(\mathrm{mg} / \mathrm{g})$} \\
\cline { 2 - 3 } Byproduct & Total phenolics & Total proanthocyanidins \\
Merlot stalk & $9.4 \pm 0.5$ & $8.2 \pm 0.2$ \\
Merlot marc & $4.9 \pm 0.1$ & $2.7 \pm 0.1$ \\
Cabernet Sauvignon & $11.6 \pm 0.5$ & $9.8 \pm 0.2$ \\
marc & $47.6 \pm 3.5$ & $24.2 \pm 0.1$ \\
Merlot leaf &
\end{tabular}




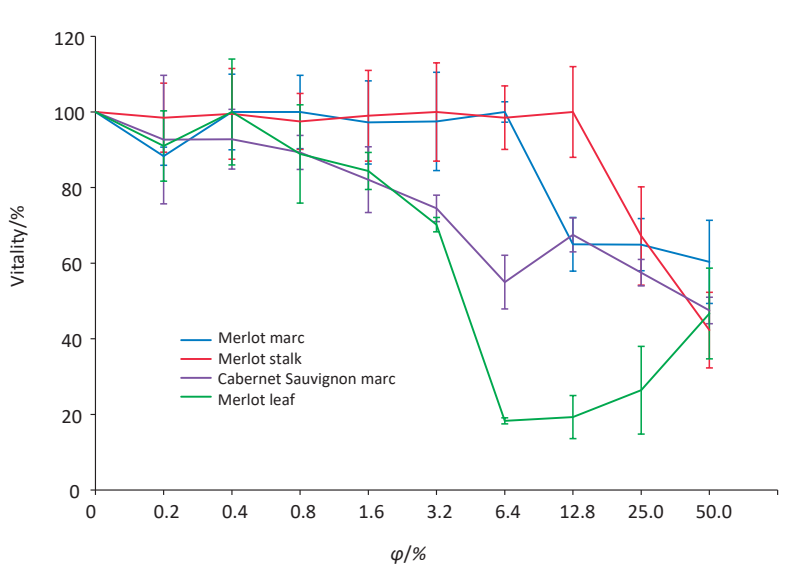

Fig. 1. Vitality (mean value \pm standard deviation, $N=3$ ) of cultured human dermal fibroblasts treated for $24 \mathrm{~h}$ with increasing volume fractions of four aqueous phytocomplexes extracted from byproducts of Vitis vinifera processing as assessed by the colorimetric (MTT) test

Results of the MTT test (Fig. 1) evidenced variable cytotoxicity of the four extracts that caused $20 \%$ decrease of cell viability at volume fractions starting from 3.2\% (Cabernet Sauvignon marc and leaves). For the two Merlot stalk and marc extracts, cell viability did not decrease more than $20 \%$ compared to control before reaching volume fractions of 25 and $12.5 \%$, respectively. According to these results, the two (low and high) non-toxic doses of extracts were set, respectively, as follows: Merlot stalks 6.4 and $12.8 \%$, Merlot marc 3.2 and $6.4 \%$, Cabernet Sauvignon marc 0.8 and $1.6 \%$, and Merlot leaves 0.8 and $1.6 \%$.

\section{Effects of extracts on cultured cells}

Plasmatic membrane status

The assay did not evidence any harmful effects of the extracts on cell membranes, while the positive controls treated with the detergent at the two volume fractions of 1 and $3 \%$ showed significant $(p<0.001)$ reduction in the percentage of fibroblasts with intact plasma membranes ((52.1 \pm 3.1$)$ and (14.5 \pm 1.7$) \%)$, respectively, compared to control ((95.2 \pm 1.2$) \%)$; data not shown.

\section{Mitochondrial transmembrane potential}

Results of the test of mitochondrial membrane potential indicated a generalized moderate decrease of mitochondrial activity after treatment with extracts of Merlot stalks and
Cabernet Sauvignon marc (Table 2). In particular, the decrease in mitochondrial activity became statistically significant at higher doses of extracts and $\mathrm{H}_{2} \mathrm{O}_{2}$ concentration of 1.5 and 4.5 $\mathrm{mM}$, while higher dose of stalk extract reduced mitochondrial activity even in the absence of $\mathrm{H}_{2} \mathrm{O}_{2}$ treatment, thus indicating an intrinsic basic level of toxicity to mitochondrial membranes. Conversely, the extracts of Merlot marc moderately increased the mitochondrial activity of fibroblasts, though not statistically significantly, in response to $\mathrm{H}_{2} \mathrm{O}_{2}$ at concentrations of 0.5 and $1.5 \mathrm{mM}$, while the extracts of leaves significantly increased the percentage of fibroblasts with active polarized mitochondrial membranes following treatment with $0.5 \mathrm{mM} \mathrm{H}_{2} \mathrm{O}_{2}$ at both the low and high volume fractions.

\section{Intracellular ROS}

The extracts of Merlot stalks did not have any statistically relevant effects on the cellular oxidative status (Table 3 ). Medium $(p<0.05)$ to highly $(p<0.001)$ positive effects of the other three extracts of marc and leaves were observable following exposure to almost all the dosages of $\mathrm{H}_{2} \mathrm{O}_{2}$. The extract of Merlot leaves showed to be the most relevant protective agent at both low and high doses even at the highest $\mathrm{H}_{2} \mathrm{O}_{2}$ concentration of $4.5 \mathrm{mM}$.

The processing of grapevine generates large quantities of byproducts. Thus, the exploitation of these wastes of high nutritional value has gained increasing interest (11). In particular, grape byproducts are very rich in secondary metabolites produced by plants in response to stress. Polyphenols, whose composition varies among pomace, skin and seeds, represent about $70 \%$ of the bioactive compounds found in fruit, but considerable concentrations are still present in the byproducts after the industrial process of winemaking (3). The results of our work showed a total phenolic content ranging from 4.9 to $47.6 \mathrm{mg}$ per $\mathrm{g}$ of lyophilizate (Table 1). The values are compatible with those reported in the literature, which show a wide variability in phenolic content and composition attributable to innumerable variables such as parts of the plant, tissue and cultivar, extraction and purification methods, geographical distribution, and even the degree of ripeness $(12,13)$. In particular, non-negligible quantities of phenolics could be recovered from stalks, which represent the less noble part of the vine. However, in spite of the content of phenolics, the extracts did

Table 2. Human dermal fibroblasts with polarized mitochondrial membranes assessed in control and in groups treated for $24 \mathrm{~h}$ with four aqueous extracts of grape byproducts at two (low and high) non-toxic volume fractions. Measurements were performed after 30 min of treatment with increasing concentrations of hydrogen peroxide

\begin{tabular}{|c|c|c|c|c|c|c|c|c|c|}
\hline \multirow{3}{*}{$c\left(\mathrm{H}_{2} \mathrm{O}_{2}\right) / \mathrm{mM}$} & \multirow{3}{*}{ Control } & \multicolumn{8}{|c|}{$N($ fibroblast $) / \%$} \\
\hline & & \multicolumn{2}{|c|}{ Merlot stalk } & \multicolumn{2}{|c|}{ Merlot marc } & \multicolumn{2}{|c|}{ Cabernet Sauvignon marc } & \multicolumn{2}{|c|}{ Merlot leaf } \\
\hline & & low & high & low & high & low & high & low & high \\
\hline 0 & $(96.9 \pm 1.8)$ & $(94.7 \pm 1.1)$ & $(89.7 \pm 2.4)^{*}$ & $(95.3 \pm 0.9)$ & $(91.5 \pm 1.0)$ & $(97.6 \pm 0.8)$ & $(96.1 \pm 1.3)$ & $(94.6 \pm 1.6)$ & $(95.3 \pm 1.7)$ \\
\hline 0.5 & $(88.2 \pm 1.1)$ & $(90.1 \pm 1.1)$ & $(81.7 \pm 11.2)$ & $(94.8 \pm 0.7)$ & $(91.5 \pm 1.1)$ & $(95.3 \pm 0.9)$ & $(92.5 \pm 1.0)$ & $(96.3 \pm 1.8)^{*}$ & $(96.4 \pm 1.4)^{*}$ \\
\hline 1.5 & $(64.6 \pm 2.4)$ & $(51.4 \pm 6.2)$ & $(49.3 \pm 6.1)^{*}$ & $(69.1 \pm 1.5)$ & $(73.6 \pm 1.2)$ & $(61.0 \pm 6.0)$ & $(52.5 \pm 4.8)$ & $(72.5 \pm 3.2)$ & $(74.4 \pm 3.3)$ \\
\hline 3.0 & $(43.6 \pm 2.7)$ & $(36.9 \pm 4.6)$ & $(32.7 \pm 2.3)$ & $(40.5 \pm 3.4)$ & $(45.5 \pm 4.0)$ & $(35.4 \pm 5.8)$ & $(40.7 \pm 7.0)$ & $(45.0 \pm 4.0)$ & $(40.0 \pm 8.4)$ \\
\hline 4.5 & $(32.5 \pm 1.3)$ & $(22.6 \pm 1.8)$ & $(22.1 \pm 1.2)$ & $(34.1 \pm 2.5)$ & $(31.0 \pm 2.1)$ & $(23.7 \pm 0.9)$ & $(15.7 \pm 8.3)^{* *}$ & $(33.5 \pm 3.5)$ & $(29.2 \pm 2.4)$ \\
\hline
\end{tabular}

Values are expressed as mean \pm standard deviation. Values within a row are statistically different compared to the control at: ${ }^{*} p<0.05,{ }^{* *} p<0.01$ 
Table 3. Intensity of green fluorescence indicative of the level of oxidation assessed in control human dermal fibroblasts and in fibroblasts treated for $24 \mathrm{~h}$ at two (low and high) non-toxic volume fractions of aqueous extracts of grape byproducts. Measurements were performed after 30 min of treatment with increasing concentrations of hydrogen peroxide

\begin{tabular}{|c|c|c|c|c|c|c|c|c|c|}
\hline \multirow{3}{*}{$c\left(\mathrm{H}_{2} \mathrm{O}_{2}\right) / \mathrm{mM}$} & \multirow{3}{*}{ Control } & \multicolumn{8}{|c|}{ Intensity/AU } \\
\hline & & \multicolumn{2}{|c|}{ Merlot stalk } & \multicolumn{2}{|c|}{ Merlot marc } & \multicolumn{2}{|c|}{ Cabernet Sauvignon marc } & \multicolumn{2}{|c|}{ Merlot leaf } \\
\hline & & low & high & low & high & low & high & low & high \\
\hline 0 & $(22.4 \pm 6.5)$ & $(26.4 \pm 6.1)$ & $(32.2 \pm 9.6)$ & $(19.0 \pm 4.0)$ & $(23.6 \pm 2.8)$ & $(18.0 \pm 3.3)$ & $(19.9 \pm 4.1)$ & $(20.0 \pm 4.4)$ & $(20.0 \pm 3.9)$ \\
\hline 0.5 & $(27.5 \pm 5.6)$ & $(30.2 \pm 8.0)$ & $(31.6 \pm 9.5)$ & $(23.7 \pm 7.2)$ & $(22.9 \pm 7.0)$ & $(26.9(4.3)$ & $(23.2 \pm 3.4)$ & $(22.8 \pm 5.6)$ & $(21.4 \pm 1.4)$ \\
\hline 1.5 & $(68.8 \pm 4.3)$ & $(64.3 \pm 4.2)$ & $(71.2 \pm 7.0)$ & $(59.1 \pm 4.3)^{* * *}$ & $(55.0 \pm 2.8)^{* * *}$ & $(58.5 \pm 5.2)^{* *}$ & $(56.9 \pm 6.8)^{* *}$ & $(39.2 \pm 3.1)^{* * *}$ & $(38.7 \pm 5.3)^{* * *}$ \\
\hline 3.0 & $(83.8 \pm 9.0)$ & $(87.9 \pm 9.5)$ & $(86.3 \pm 7.4)$ & $(84.0 \pm 10.2)$ & $(85.2 \pm 4.4)$ & $(69.3 \pm 6.6)^{*}$ & $(67.4 \pm 6.6)^{* *}$ & $(51.7 \pm 2.6)^{* * *}$ & $(53.9 \pm 2.5)^{* * *}$ \\
\hline 4.5 & $(117.3 \pm 15.0)$ & $(108.0 \pm 4.5)$ & $(109.4 \pm 9.1)$ & $(125.3 \pm 19.9)$ & $(108.7 \pm 8.9)$ & $(96.5 \pm 2.7)^{* * *}$ & $(104.6 \pm 8.5)$ & $(81.6 \pm 4.2)^{* * *}$ & $(87.8 \pm 4.4)^{* *}$ \\
\hline
\end{tabular}

Values are expressed as mean \pm standard deviation. Values within the same row are statistically different compared to the control at: ${ }^{*} \mathrm{p}<0.05$, ${ }^{* *} \mathrm{p}<0.01,{ }^{* * *} \mathrm{p}<0.001$

not elicit significantly protective biological activity. On the contrary, these extracts showed mild signs of toxicity evidenced by decreased number of fibroblasts with polarized mitochondrial membranes at volume fractions indicated as not harmful by the MTT test. This result prompts the need for increasingly informative analytical assessments to unravel the general health state of the cell and of its cellular compartments. In this respect, the colorimetric MTT test, which is used to assess cell viability based on the enzymatic reduction of MTT to formazan by the mitochondrial succinate dehydrogenase, indirectly allows for the assessment of cellular energy. On the contrary, the assessment of mitochondrial membrane activity in cytofluorometry is a direct estimate of the functionality of the respiratory electron transport chain. Similarly, cytofluorometric evaluations offer growing investigative opportunities thanks to the multitude of fluorochromes with affinity for different analytes and cell compartments (14).

Since various classes of molecules can derive from different plant matrices, in the present work the MTT procedure, a universally accepted method for determining the dosage of chemicals/drugs causing general cellular toxicity, represented the first step for implementing the successive specific tests in cytofluorimetry using non-toxic doses of the extracts. At the same time, these 'safe' dosages also resulted in the proportioning into a more uniform range of total phenolic and proanthocyanidin mass fractions among samples. Thus, it is plausible to speculate that the observed differences among extracts cannot simply be ascribed to a mere quantity of phenols, but even more to their finest composition in valuable molecules. This is evident, for example, for extracts of Cabernet Sauvignon marc, which despite the double initial total phenolic and proanthocyanidin content, showed better results even when tested at eight times lower concentration than of Merlot. Leaf extracts demonstrated more beneficial effects at volume fractions up to 16 times lower than stalks despite having only 5 and 3 times higher total phenolic and proanthocyanidin contents, respectively. This finding should be considered in conjunction with the fact that innumerable variables, such as plant varieties, soil characteristics, sunlight exposure, pretransformation and extraction processes (drying, grinding, etc.), could be determinant for the quantity and the composition of extracts. For these reasons, customized separation techniques for individual compounds are still advisable for a large-scale application of agro-wastes for industrial purposes (15). In fact, when highly selective processes were applied to purify specific stilbenes from stalks of Vitis vinifera, these byproducts proved to still be a rich source of compounds with biological activity (16).

As for stalks, the Naviglio extractor allowed for the recovery of relevant classes of molecules from the marc and leaves, which were able to exert variable protective antioxidant effects to fibroblasts. In particular, leaf extracts showed significant protective effects also on mitochondrial membranes at $0.5 \mathrm{mM} \mathrm{H}_{2} \mathrm{O}_{2}$ and moderate effects at $1.5 \mathrm{mM} \mathrm{H}_{2} \mathrm{O}_{2}$, although not to a significant level. Similar beneficial effects were also shown by the two marc extracts and in particularly by that of Cabernet Sauvignon. In this respect, increasing numbers of studies are focusing on the characterization of the chemical profile of extracts among Vitis vinifera varieties. Within these, Anđelković et al. (17) have shown that, more than the variety, the health status of the plant is more likely to influence the synthesis of specific secondary metabolites as a defense against infections. Oppositely, as reported by other authors $(18,19)$, the phenolic content and composition of the leaf and grape extracts appeared to be largely cultivar and season-dependent. However, similar studies on grape pomace are limited (20), despite its large quantity produced by winemaking industry. Therefore, with respect to our present work, it is possible to speculate that higher antioxidant properties of the extracts of Cabernet Sauvignon marc than those of Merlot variety can be attributable both to the higher content and quality of its phenolic acids and proanthocyanidins (21).

Several molecules with both beneficial or adverse effects can derive from plants (22), based on the nature of the extracts (e.g. crude or solvent fraction) and the part of the plant of origin (e.g. root, stem or leaf). For this reason, in the present work the extracts were specifically tested for their safety against cell membranes. In this respect, in the cosmetics in general, there is the strong need to combine the product functionality with the maintenance of the structure of cell membranes of epidermal tissues to which the product is intended. As the result, none of the extracts showed adverse effects to plasmatic membranes. In our opinion, this result was not obvious because, despite the reassuring literature on the safety of products deriving from the vine $(1,3,6,11-13,16,21)$, important classes of molecules with potential biological hazards, such as haemolytic effects, have not been studied extensively (23). 
Extremely variable ranges of temperatures and solvents have been used to retrieve active molecules from vine plant with yields depending on the matrix, the polar nature of the extract, or even on the plant variety (red or white) (24). The selective recovery of particular components requires fine setting of the critical conditions, while the choice of the most suitable solvent compatible with requirements of the final cosmetic/food/pharma user industry is a major constrain. In this regard, the dynamic Naviglio extraction method with water at low temperature $\left(25-27^{\circ} \mathrm{C}\right)$ has already achieved extraction yields comparable to conventional solid-liquid extraction with boiling water, and even superior to both microwave and pressurized solvent extractions of phenolic, volatile and mineral compounds from vine waste with water at $100^{\circ} \mathrm{C}(25)$.

\section{CONCLUSIONS}

This work demonstrated the beneficial effects of aqueous extracts at low temperature from vine waste products by using cytofluorimetry analytical method and cultured human dermal fibroblasts. This result is relevant in view of the increasing interest towards agricultural derivatives as attractive sources of low-cost active ingredients for several applications. In particular, due to the low operating temperatures, the Naviglio technology represents an environmentally friendly approach to replace organic solvents with water, particularly intended for preservation of thermolabile compounds. On the other hand, when non-selective extraction methods are used, detailed screening of the extracts is recommended in order to avoid underestimation of possible cytotoxic effects of secondary metabolites. The tests based on cytofluorometric screening of cellular and mitochondrial membranes represent a valid compromise between the evaluation of cytotoxic and protective effects based on highly versatile procedures.

\section{FUNDING}

This work was funded by the projects '2014-1729' and 'ID $60209936^{\prime}$ of the CARIPLO Foundation, Milan, and Lombardy Region, Italy, respectively.

\section{REFERENCES}

1. Nassiri-Asl M, Hosseinzadeh H. Review of the pharmacological effects of Vitis vinifera (grape) and its bioactive compounds. Phytother Res. 2009;23(9):1197-204.

https://doi.org/10.1002/ptr.2761

2. Domínguez J, Martínez-Cordeiro H, Álvarez-Casas M, Lores M. Vermicomposting grape marc yields high quality organic biofertiliser and bioactive polyphenols. Waste Manage Res. 2014;32(12):1235-40. https://doi.org/10.1177/0734242X14555805

3. Mattos GN, Tonon RV, Furtado AA, Cabral LMC. Grape by-product extracts against microbial proliferation and lipid oxidation: a review. J Sci Food Agric. 2017;97(4):1055-64. https://doi.org/10.1002/jsfa.8062
4. Daneshian M, Akbarsha MA, Blaauboer B, Caloni F, Cosson $P$, Curren $R$, et al. A framework program for the teaching of alternative methods (replacement, reduction, refinement) to animal experimentation. ALTEX. 2011;28(4):341-52.

https://doi.org/10.14573/altex.2011.4.341

5. Adan A, Alizada G, Kiraz Y, Baran Y, Nalbant A. Flow cytometry: basic principles and applications. Crit Rev Biotechnol. 2017;37(2):163-76.

https://doi.org/10.3109/07388551.2015.1128876

6. Fontana AR, Antoniolli A, Bottini R. Grape pomace as a sustainable source of bioactive compounds: extraction, characterization, and biotechnological applications of phenolics. J Agric Food Chem. 2013;61(38):8987-9003. https://doi.org/10.1021/jf402586f

7. Naviglio D. Naviglio's principle and presentation of an innovative solid-liquid extraction technology: Extractor Naviglio ${ }^{\oplus}$. Anal Lett. 2003;36(8):1647-59.

https://doi.org/10.1081/AL-120021555

8. Singleton VL, Rossi JA. Colorimetry of total phenolics with phosphomolybdic-pfosphotungstic acid reagents. Am J Enol Vitic. 1965;16:144-58.

9. Porter LJ, Hristich LN, Chan BG. The conversion of procyanidins and prodelphinidins to cyanidin and delphinidin. Phytochemistry. 1985;25:223-30.

https://doi.org/10.1016/S0031-9422(00)94533-3

10. Puglisi R, Bornaghi V, Severgnini A, Vanni R, Balduzzi D, Galli A. Cryopreservation of stallion semen: laboratory assessment of sperm injuries after cushioned centrifugation and freezing with conventional and alternative directional freezing methods. Jpn J Vet Res. 2016;64(4):235-45.

https://doi.org/10.14943/jjvr.64.4.235

11. Djilas S, Čanadanović-Brunet J, Ćetković G. By-products of fruits processing as a source of phytochemicals. Chem Ind Chem Eng Q. 2009;15(4):191-202.

https://doi.org/10.2298/ciceq0904191d

12. Monagas M, Hernández-Ledesma B, Gómez-Cordovés $C$, Bartolomé B. Commercial dietary ingredients from Vitis vinifera L. leaves and grape skins: Antioxidant and chemical characterization. J Agric Food Chem. 2006;54(2):319-27. https://doi.org/10.1021/jf051807j

13. Farhadi K, Esmaeilzadeh F, Hatami M, Forough M, Molaie R. Determination of phenolic compounds content and antioxidant activity in skin, pulp, seed, cane and leaf of five native grape cultivars in West Azerbaijan province, Iran. Food Chem. 2016;199:847-55.

https://doi.org/10.1016/j.foodchem.2015.12.083

14. Shapiro HM. Flow cytometry: the glass is half full. In: Hawley T, Hawley R, editors. Flow cytometry protocols. Methods in molecular biology, vol. 1678. New York, NY, USA: Humana Press; 2018; pp. 1-10.

https://doi.org/10.1007/978-1-4939-7346-0_1

15. Yammine S, Brianceau S, Manteau S, Turk M, Ghidossi R, Vorobiev E, Mietton-Peuchot M. Extraction and purification of 
high added value compounds from by-products of the winemaking chain using alternative/nonconventional processes/technologies. Crit Rev Food Sci Nutr. 2018;58(8):1375-90. https://doi.org/10.1080/10408398.2016.1259982

16. Papastamoulis Y, Richard T, Nassra M, Badoc A, Krisa S, Harakat $D$, et al. Viniphenol $A$, a complex resveratrol hexamer from Vitis vinifera stalks: Structural elucidation and protective effects against amyloid- $\beta$-induced toxicity in PC1 2 cells. J Nat Prod. 2014;77(2):213-7. https://doi.org/10.1021/np4005294

17. Anđelković $M$, Radovanović $B$, Milenkovic Anđelković $A$, Radovanović V. Phenolic compounds and bioactivity of healthy and infected grapevine leaf extracts from red varieties Merlot and Vranac (Vitis vinifera L.). Plant Foods Hum Nutr. 2015;70(3):317-23.

https://doi.org/10.1007/s11130-015-0496-3

18. Serrano A, Espinoza C, Armijo G, Inostroza-Blancheteau C, Poblete E, Meyer-Regueiro C, et al. Omics approaches for understanding grapevine berry development: Regulatory networks associated with endogenous processes and environmental responses. Front Plant Sci. 2017;8:Article no. 1486.

https://doi.org/10.3389/fpls.2017.01486

19. Correia AC, Jordão AM. Antioxidant capacity, radical scavenger activity, lipid oxidation protection analysis and antimicrobial activity of red grape extracts from different varieties cultivated in Portugal. Nat Prod Res. 2015;29(5):438-40. https://doi.org/10.1080/14786419.2014.945086

20. de la Cerda-Carrasco A, López-Solís R, Nuñez-Kalasic H, Peña-Neira Á, Obreque-Slier E. Phenolic composition and antioxidant capacity of pomaces from four grape varieties (Vitis vinifera L.). J Sci Food Agric. 2015;95(7):1521-7. https://doi.org/10.1002/jsfa.6856

21. Shankar E, Goel A, Gupta K, Gupta S. Plant flavone apigenin: an emerging anticancer agent. Curr Pharmacol Rep. 2017;3(6):423-46.

https://doi.org/10.1007/s40495-017-0113-2

22. Top H, Sarikahya NB, Nalbantsoy A, Kirmizigul S. Immunomodulatory, hemolytic properties and cytotoxic activity potent of triterpenoid saponins from Cephalaria balansae. Phytochemistry. 2017;137:139-47.

https://doi.org/10.1016/j.phytochem.2017.02.015

23. Pensec F, Szakiel A, Paczkowski C, Wozniak A, Grabarczyk M, Bertsch C, et al. Characterization of triterpenoid profiles and triterpene synthase expression in the leaves of eight Vitis vinifera cultivars grown in the Upper Rhine Valley. J Plant Res. 2016,129(3):499-512.

https://doi.org/10.1007/s10265-016-0797-0

24. Domínguez-Perles R, Teixeira Al, Rosa E, Barros Al. Assessment of (poly)phenols in grape (Vitis vinifera L.) stems by using food/pharma industry compatible solvents and response surface methodology. Food Chem. 2014;164:33946.

https://doi.org/10.1016/j.foodchem.2014.05.020

25. Sánchez-Gómez R, Zalacain A, Alonso GL, Salinas MR. Vine-shoot waste aqueous extracts for re-use in agriculture obtained by different extraction techniques: phenolic, volatile, and mineral compounds. J Agric Food Chem. 2014;62(45):10861-72.

https://doi.org/10.1021/jf503929v 